ARCHIVO ESPAÑOL DE ARTE, LXXXVIII, 352

OCTUBRE-DICIEMBRE 2015, pp. 409-417

ISSN: 0004-0428, eISSN: 1988-8511

doi: $10.3989 /$ aearte.2015.26

\title{
LOS “ESTIGMAS” DEL GRECO EN ROMA. A PROPÓSITO DE UNA OBRA PERDIDA DEL GRECO
}

\author{
Panayotis K. IOANNOU \\ Universidad de Creta - Instituto de Estudios Mediterráneos
}

\begin{abstract}
Según un antiguo testimonio, incluido en la obra de Seroux D'Agincourt, existía, al menos a finales del siglo XVIII, un San Francisco de El Greco en la iglesia de San Rocco en Roma. En este artículo se presenta y examina dicha información contrastada con las fuentes, documentación de archivo, y obras conocidas de El Greco.

Palabras clave: El Greco; Domenicos Theotocopoulos; Imagen de S. Francisco; Iglesia de San Rocco; Roma; Ignacio Zuloaga; Rocco Pagliara.
\end{abstract}

\section{THE STIGMATA OF EL GRECO IN ROME: ON A LOST PAINTING BY THEOTOCOPOULOS}

According to an old testimony cited by Seroux D'Agincourt, in the late $18^{\text {th }}$ century there was a painting of Saint Francis by El Greco in the Roman church of San Rocco. The author investigates this testimony in the light of source materials, archival documentation and the known works of El Greco.

Key words: El Greco; Domenicos Theotocopoulos; Image of S. Francis; Church of San Rocco; Rome; Ignacio Zuloaga; Rocco Pagliara.

Jean Baptiste Louis Georges Séroux D’Agincourt (1730-1814), en su obra fundamental sobre las artes figurativas entre "decadencia" y "renovación", calificaba la producción pictórica de un vagamente denominado arte griego medieval, relacionado con el arte italiano, como: "vieille école grecque en Grèce / Xe, XIe, XIIe et XIIIe siècles", "école mixte, gréco-italienne / XIIe et XIIIe siècles" y "école d'imitation". Séroux D’Agincourt aportaba documentación procedente de investigaciones en archivo, monumentos y colecciones, y de la densa correspondencia que mantenía con un amplio círculo de literatos, escritores, historiadores y anticuarios de toda Europa, sobre todo de la península italiana, donde se había instalado en 1782, cuando concibió la idea de redactar su extenso libro'. El escritor francés, en una lista titulada "maîtres d'une école mixte" [de los siglos XIII-XIV], incluida en el capítulo del mismo nombre, recogía la siguiente noticia, de procedencia indirecta:

"Dominique Theoscopolis. - tableau représentant St François d'Assise, exposé au XVe siècle, près de l'église de St Roch, à Rome; avec cette inscription en caractères grecs, $\triangle \mathrm{OMHNIKO \Sigma} \Theta \mathrm{OE}-$ $\mathrm{O} \Sigma \mathrm{KO \Pi O} \Lambda \mathrm{I} \Sigma \mathrm{E}$. Notice, par Amaduzzi, professeur de grec, à la Sapience, à Rome"3.

\footnotetext{
${ }^{1}$ Séroux D’Agincourt, 1823: 87-104. Sobre el enfoque de Séroux d'Agincourt en relación con el arte griego que ve "sofocado" bajo la iglesia bizantina, véase los breves comentarios de Haskell, 1989: 38-39.

2 Sobre la obra di Séroux d'Agincourt véase, entre la amplia bibliografía Pommier, 1997: 27-35. Griener, 1997. Berchet, 1999. Miarelli Mariani, 2002; 2005. Mondini, 2005.

${ }^{3}$ Séroux D'Agincourt, 1823: 100, nota (la cursiva es del original).
} 
Rápidamente advertimos que, si tenemos en cuenta lo que hasta ahora sabemos sobre la actividad y obra del Greco, no se conoce ningún cuadro suyo en la iglesia romana de San Rocco o en la «Escuela de san Rocco», tal y como se recoge en la edición italiana del libro de Seroux D’Agincourt ("Quadro rappresentante S. Francesco d'Assisi nella Scuola di San Rocco, a Roma") ${ }^{4}$. Debemos señalar también que durante nuestra investigación en la iglesia tampoco hemos encontrado ningún cuadro que pudiera identificarse con la obra que se citaba en la Histoire de l'art. Sin embargo, no se debe por ello dudar de la fidelidad de dicha noticia: hacia finales del siglo XVIII, cuando suponemos que le llegó la noticia del cuadro a Séroux d'Agincourt -seguramente antes de la muerte de Amaduzzi, en 1792- el nombre del pintor cretense era desconocido en el ámbito italiano, incluso era confundido con el de otros artistas; es más, es muy probable que nadie quisiera dar una falsa noticia sobre una obra del Greco. Incluso es posible que Giancristofano Amaduzzi, profesor de griego en la Universidad de Roma y personaje destacado en el siglo XVIII italiano ${ }^{5}$, no conociera siquiera la existencia de este pintor. Aunque el nombre del artista cretense había aparecido ya en el Abcedario Pittorico de Orlandi, en la edición de 1753 como "Domenico Teoscopoli", parece que Séroux d'Agincourt añadió el «Theoscopolis» en la lista de la école mixte, basándose solo en la lectura de la inscripción del cuadro, puesto que no citó bibliografía alguna. Hay que señalar también que la firma del artista, tal y como la transcribe, erróneamente, Amaduzzi (" $\Delta \mathrm{OMHNIKO \Sigma} \Theta \mathrm{EO} \Sigma \mathrm{KO} \mathrm{O} \Lambda \mathrm{I} \Sigma \mathrm{E} \Pi$. [ ]"), puede identificarse con una de las maneras que el pintor usaba para firmar sus obras: $\triangle$ OMHNIKO $\Sigma$ OEOTOKOПOY $\Lambda$ O $\Sigma$ EПOIEI.

La noticia sobre la existencia del mencionado cuadro, que -a diferencia de otras muchas obras incluidas en el mismo volumen de Séroux d'Agincourt- no está ilustrado, no fue comprobada en ediciones posteriores, ni en las traducciones comentadas italianas, y parece que ha pasado desapercibida para toda la bibliografía theotocopuliana.

Obviamente, el cuadro no podía estar expuesto en la iglesia de San Rocco en el siglo XV, no solo porque en ese caso sería imposible que la obra fuera de mano de Theocopoulos, sino porque además las obras de construcción de la iglesia y del hospital de esta homónima confraternidad no comenzaron antes del siglo $\mathrm{XVI}^{7}$. Por lo tanto, esta referencia cronológica se debe, bien a un error tipográfico, o bien es fruto de la observación estilística de Séroux D'Agincourt que, en general, fechaba las obras de los artistas de origen griego, atribuyendo sus reminiscencias medievales a un periodo precedente a la "renovación de las artes". Así "Theoscopolis", como artista "griego", junto con Andrea Rico, los pintores Bizamani y otros, con los que aparecía dentro de la misma "escuela", era considerado como un pintor "primitivo".

En resumen, el cuadro firmado por el Greco debería sin duda, estar expuesto en San Rocco, no se sabe dónde, al menos a finales del siglo XVIII, cuando Amaduzzi leyó la primera firma-inscripción. Sin embargo, no hemos podido encontrar ningún testimonio de la obra en las publicaciones sobre el complejo monumental de San Rocco, ni siquiera en las guías de la ciudad de Roma

\footnotetext{
${ }^{4}$ Séroux d'Agincourt 1835: 179. cfr. también la referencia del griego Andreas Mustoxidis (Mustoxidis, 1843: 272). En 1855, el griego S.N. Kaloutsis, repite la información transmitida por Séroux d'Agincourt y aporta ya el nombre

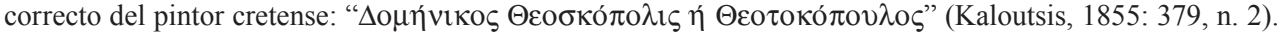

${ }^{5}$ Sobre Amaduzzi véase Fabi, 1961. Caffiero, 1974; 1976.

${ }^{6}$ Orlandi, 1753: 149. Todavía en 1831, Giovanni Pagano, en la guía del Museo de Nápoles, refiriéndose al autor del retrato de Giulio Clovio, catalogaba el cuadro como "Domenico Teoscopuli, ovvero Domenico, detto delle Greche", ignorando la precisión ya hecha por Pietro Zani (Zani, 1822: 172). El mismo Pagano escribió que «in Italia non si conosce con certezza verun'opera pittorica di questo Teoscopoli» (Pagano, 1831: 83-84, n. 211).

${ }^{7}$ Sobre la iglesia de San Rocco véase Salerno/Spagnesi, 1962.

${ }^{8}$ Sobre las fechas de las obras consideradas "medievales", en las que se incluían brevemente todos los pintores cuyo estilo parecía anterior al renacimiento, véase la obra básica de Previtali, 1989.
}

Arch. esp. arte, LXXXVIII, 352, OCTUBRE-DICIEMBRE 2015, 409-417 ISSN: 0004-0428, eISSN: 1988-8511, doi: 10.3989/aearte.2015.26 
publicadas en los siglos XVII y XVIII ${ }^{9}$. En cambio, en la investigación de archivo, los resultados fueron más prometedores, aunque no los deseados.

Según los inventarios del siglo XIX de los muebles de la iglesia y de la confraternidad de San Rocco - conservados en el Archivo del Vicariato de Roma-, en el "vestiario grande", lugar que no es fácil identificar hoy, se registra "un cuadro con marco dorado que representa San Francisco, de buena mano" (inventarios redactados en 1866 y 1869) ${ }^{10}$. Del mismo modo, según otro inventario del siglo XVIII, conservado en el Archivo de Estado de Roma, en la sacristía de San Rocco, construida en $1651^{11}$, existía un cuadrito que representaba a San Francisco con "marco dorado"12.

No se puede saber si estas descripciones tan genéricas corresponden o no con la obra citada en el libro de Séroux d'Agincourt; menos aún las breves referencias a "cuadros" de pequeño tamaño que se encontraban en la sacristía, en el "vestiario grande" y en el oratorio identificable con la "Escuela"13, aunque en estos casos no se precisa siquiera el sujeto representado (inventarios fechados en $1827^{14}$, en $1829^{15}$, en $1834^{16}$, en $1864^{17}, 1866^{18}$ y $1869^{19}$ ).

La iglesia y el "oratorio" sufrieron una drástica transformación durante las primeras décadas del siglo XX. Entre 1909 y 1910 se llevaron a cabo trabajos de restauración en la iglesia; en 1934, con motivo de la promoción del adyacente Mausoleo de Augusto, se demolieron tanto el Oratorio como el hospital de San Rocco ${ }^{20}$. No se tiene ninguna noticia de la pintura de San Francisco que habría ingresado en la confraternidad, no se sabe cuándo, tal vez, a través de alguna donación, dadas las pequeñas dimensiones del cuadro, si es que podemos identificar esta pintura con uno de los cuadritos citados en los inventarios.

\footnotetext{
${ }^{9}$ En la guías de este tipo no se incluyen descripciones breves de las obras en el interior de la iglesia de San Rocco; véase, a título indicativo, Descrittione di Roma antica e moderna, Roma, Andrea Fei, 1643: 90-91. Titi, 1675: 239-240; 1686: 364-365; 1763: 394-395. Posterla, 1707: 458-461. Venuti, 1767: 401-404.

${ }^{10}$ Inventario e consegna degli oggetti esistenti nella Provveditoria di chiesa della Ven. Arciconfraternita di S. Rocco, Archivio del Vicariato, Roma (en andelante AVR), Confraternite ed Arciconfraternite: Arciconfraternita di San Rocco, vol. 89 , inventario suelto, n. 51.

${ }^{11}$ Salerno/Spagnesi, 1962: 60, 74

12 Salerno/Spagnesi, 1962: 77.

${ }^{13}$ Para las pinturas conservadas en el Hospital véase también Garofalo, 1949: 31 (se describe el Oratorio de San Rocco como una "piccola aula rettangolare, probabilmente di scarso interesse architettonico, decorata prevalentemente da un certo numero di quadri, che poi furono trasferiti nella chiesa").

14 "Quattro quadri che stavano in sagrestia" (AVR, Arciconfraternita di San Rocco, vol. 89, inventario suelto, 1827, n. 5).

15 "4 quadri usati portativi dalla sagrestia" [en el Vestiario Grande] y "due altri [cuadros] piccoli" (AVR, Arciconfraternita di San Rocco, vol. 89, inventario suelto, 1829, nn. 86 y 165).

16 "Quattro tavolette alle finestre dell'Oratorio con rispettive tele di buono stato" y "due altri [quadri] piccoli" (AVR, Arciconfraternita di San Rocco, vol. 89, inventario suelto, 1834, nn. 8 y 66).

17 "Quattro tavolette alle fenestre dell'Oratorio con respettive tele in cattivo stato" y "quattro quadri usati portati dalla sagrestia" (AVR, Arciconfraternita di San Rocco, vol. 89, inventario suelto del 1864, nn. 9 y 91).

18 "Quattro tavolette alle finestre dell'Oratorio con rispettive tele a buono stato" (AVR, Arciconfraternita di San Rocco, inventario suelto, 1866, n. 9).

19 "Quattro tavolette alle finestre dell'Oratorio con respettive tele di buono stato" (AVR, Arciconfraternita di San Rocco, inventario suelto, 1869, n. 9). Es muy probable que todas estas obras se refieran a una imagen de San Francisco de Asís y no a la de San Francisco de Paula que se representa en la pala de altar y no puede calificarse de "quadretto". El cuadro de San Francesco de Paula, del interior de la iglesia está firmado por Antonio Amorosi y fechado en 1719 (Salerno/Spagnesi, 1962: 61-62, 75, fig. XLV).

${ }^{20}$ Véase al respecto Salerno/Spagnesi, 1962: 18-19. Quizás sea inútil señalar que en los primeros años del siglo XX la glesia de San Roco - cerrada desde 1904 porque era peligrosa, estaba "spogliata dei suoi averi uno dopo l'altro" (Salerno/Spagnesi, 1962: 18: "Mentre l'officiatura veniva trasferita nella vicina chiesa di San Girolamo degli Illirici, l'allora parroco di San Rocco, Monsignor Filippo Franceschini, dovette affrontare il problema di reperire i fondi per i restauri; ciò che era in effetti difficile, essendo stata la chiesa spogliata dei suoi averi uno dopo l'altro").
}

Arch. esp. arte, LXXXVIII, 352, OCTUBRE-DICIEMBRE 2015, 409-417 ISSN: 0004-0428, eISSN: 1988-8511, doi: 10.3989/aearte.2015.26 
Abandonado, de momento, todo intento de localizar algún dato sobre la que sería la única obra conocida de El Greco expuesta en una iglesia italiana, intentaremos ver si esta pintura, todavía ilocalizada, podría identificarse con alguno de los San Francisco conocidos del pintor cretense, firmados y realizados durante su estancia romana, entre 1570 y $1576^{21}$.

Partimos de dos ideas preliminares: 1) el tipo de la firma $\triangle O M H N I K O \Sigma \Theta E O T O K O П O$ $\mathrm{Y} \Lambda \mathrm{O} \Sigma \mathrm{E \Pi OIEI} \mathrm{lo} \mathrm{pone} \mathrm{en} \mathrm{relación} \mathrm{con} \mathrm{las} \mathrm{obras} \mathrm{que} \mathrm{el} \mathrm{pintor} \mathrm{realizó} \mathrm{durante} \mathrm{su} \mathrm{periodo} \mathrm{de} \mathrm{ac-}$ tividad italiana, especialmente romana ${ }^{22}$; 2) si bien, aunque en la información que da Amaduzzi no se especifica el sujeto de la pintura, hay que señalar que durante su estancia en la península italiana, el Greco se ocupó solo de la iconografía de San Francisco de Asís que recibe los estigmas. De esta iconografía franciscana, solo dos cuadros, firmados por el Greco y datados en el periodo romano, se pueden citar con certeza: un San Francisco que recibe los estigmas, en la colección de Ignacio Zuloaga y otro casi idéntico, actualmente en el Instituto "Suor Orsola Benincasa" de Nápoles; existe una tercera obra, en la Academia Carrara de Bérgamo, atribuida al Greco por Corrado Ricci en 1913, pero que no posee firma alguna del $\operatorname{artista}^{23}$. Es muy posible que ninguna de estas obras guarde la mínima relación con la pintura expuesta en San Rocco. Sin embargo, tal vez no sea del todo inútil hacer algunas consideraciones al respecto.

La obra que pertenecía a Ignacio Zuloaga (1870-1945), pintor español que, como es sabido, fue uno de los grandes admiradores, además de coleccionista de obras del pintor griego ${ }^{24}$, aparece citada contemporáneamente por primera vez, en 1908, por Manuel Bartolomé Cossío y por Paul Lafond $^{25}$. Cossío catalogaba la pintura (temple sobre tabla, 29 × $21 \mathrm{~cm}$ ) en la colección de Zuloaga en París y la fechaba en el periodo de "transición de Italia a España, 1576-1579". El escritor señalaba además que la pintura procedía de la colección Salazar en Bilbao ${ }^{26}$. En 1912, Maurice Barrès publicaba el cuadro también como perteneciente a la colección parisina de Zuloaga ${ }^{27}$. Allí lo cita todavía Mayer en 1916, quien esta vez lo fechaba en el periodo romano del Greco ("15731576 " ${ }^{28}$. Continúan las referencias a la obra en la colección Zuloaga y casi siempre fechadas en el periodo romano del $\mathrm{Greco}^{29}$. Sin embargo, aunque la pintura aparecía publicada, e incluso a

${ }^{21}$ Como es sabido, durante toda su actividad, El Greco pintó numeroso cuadros de San Francisco de Asís, ya sea recibiendo los estigmas, ya sea en éxtasis, hasta el punto de que Pacheco calificó al Cretense como "el mejor pintor deste Santo que se hubiera conocido en este tiempo" (Pacheco, 1990: 698), cfr. también Galavaris, 1995. Wismer/ScholzHänsel, 2012: 52-55, 74-79).

${ }^{22}$ Chatzidakis, 1995: 152.

${ }^{23}$ Ricci, 1913: 309-314. Sobre la obra de Bérgamo véase Pallucchini, 1981: 256. Álvarez Lopera, 2007: 87-88. Wismer/Scholz-Hänsel, 2012: 52-54. Marías, 2014: 76-77.

${ }^{24}$ A mitad del segundo decenio del siglo XIX, en el taller parisino del pintor existían "several more Grecos -Greco being, according to him, «il Dios de la pintura»" (Sargent, 1916: 32. Marías, 2009: 321). Sobre Zuloaga como coleccionista de obras del Greco véase Marías, 2009. Storm, 2011: 95-102. Thiemann, 2012.

${ }^{25}$ Lafond, 1908: 25 (ill.). En su libro sobre el Greco, de 1910, Lafond también se refiere a la obra Amor Sagrado y Profano [La apertura del Quinto Sello] entonces en la colección Zuloaga (Lafond, 1910: 68). Escribe todavía: "[sobre las imágenes de San Francisco] en Paris, chez M. Christian Cherfils, chez le peintre Ignacio Zuloaga" (Lafond, 1913: 71, cfr. también: 72-73, VII, lam. XIV).

${ }^{26}$ Cossío, 1908: 603, n. 326: "Paris. D. Ignacio Zuloaga (54, rue Caulaincourt). San Francisco de Asis, ¿Ia epoca. 1577?, - 0,29 x 0,21. - Tabla V, páginas 101 y 373; lam. 93. Firmado. Adquirido, en Bilbao, del Sr. Nardiz, procedente de la colección Salazar". Cfr. también Marías, 2009: 22-23.

${ }^{27}$ Barrès, 1912: 151-152, ilustrado (Photo Duet). En 1923, el propio Barrès cita todavía la pintura en la colección Zuloaga (Barrès, 1923: 131-132, no ilustrado).

${ }_{28}^{28}$ Mayer, 1916: 59 (no ilustrado).

${ }^{29}$ Willumsen, 1927, I: 429-440 (ilustrado). Mauclair, 1931: 9 (ilustrado). Magnoni, 1931: 72 (“S. Francesco, 15901599, Coll. Zuloaga, Parigi”). Cassou, 1931: 18, lam. 20 (ilustrado, foto: Moreno). Legendre/Hartmann, 1937: 432 («Ignacio Zuloaga, Zymaya. Photo Druet, Paris»). Escholier, 1937: 23. Pfister, 1941: 148 (“Zumaya, Sammlung D. Ignacio Zuloaga"), ilustrado en la página 29. 
veces reproducida en blanco negro a principios del siglo XX, nunca se expuso en público, salvo en la Secesión vienesa en $1903^{30}$. Wethey, en 1967, catalogaba el cuadro, y lo fechaba "ca. 1560", pero sin haberlo visto, solo a través de una fotografía, en la colección de Antonio Zuolaga en Ginebra $^{31}$. A pesar de la insistente búsqueda llevada a cabo por Nicos Hadjinicolaou desde 1995, no se había podido localizar la pintura ${ }^{32}$. Posteriormente, en 2003, la obra reaparecía en Nueva York en la exposición dedicada al artista cretense, como perteneciente a una colección privada. Por su parte, Lopera, que catalogaba el cuadro "ca. 1570-1572", recapitulando su fortuna, se refería a él como parte de la colección de los herederos de Zuloaga en Suiza, y comentaba además las imprecisiones de Cossío acerca de la procedencia de la pintura antes de que la adquiriera Zuolaga "desde antes de $1907^{\prime 33}$.

La pintura napolitana, temple sobre tabla, $(28,3 \times 20,4 \mathrm{~cm})$, firmada a bajo izquierda, en caracteres griegos " $\triangle \mathrm{OMHNIKO} \Sigma$ $\Theta \mathrm{EOTOKOПOY} \Lambda \mathrm{O} \Sigma$ EПOIEI", firma apenas visible todavía existente, se dio a conocer en 1923, en una breve nota en el periódico Napoli Nobilissima, como obra de la producción italiana tardía del Greco. El escritor anónimo (¿Aldo De Rinaldis?), al informar de la existencia del cuadro, consideró útil añadir además que la obra, que entonces estaba "en casa de las señoritas Antonietta y Adelaide Pagliara", fue comprada por Rocco Pagliara, "hace varios años a un chamarilero en Nápoles". El escritor señalaba además que el coleccionista napolitano "prestó aquel cuadrito al pintor Ignacio Zuloaga, que se lo llevó a París, donde le quitaron la suciedad y algunos viejos barnices oxidados que lo ocultaban. Una vez muerto Pagliara, el pintor español lo devolvió a sus herederos que todavía lo conservan". El autor de esta nota, después de precisar que los hechos ocurrieron "hace cinco o seis años", señalaba que "Maurizio Barrès, en su libro Greco ou le secret de Tolède, publicó una foto del cuadrito Pagliara como obra perteneciente a la colección Zuloaga de París", añadiendo que "Mayer da noticia, hoy, de un San Francisco que recibe los estigmas del Greco en la misma colección parisina". Al final, el autor se preguntaba si "el cuadro citado por Mayer es el mismo que el publicado por Barrés (perteneciente a Pagliara) o ¿se trata de otra pintura del Greco del mismo asunto?"34.

Es evidente, por las menciones a Barrès y Mayer $^{35}$ y a sus ediciones respectivas de 1912 y 1916 (no ilustrada), que el citado autor anónimo conocía la existencia de una pintura en la colección Zuloaga. Es más, sostiene repetidas veces que la foto publicada por Barrés correspondía con la obra de Pagliara, prestada por Zuloaga, sobreentendiendo que el autor del Greco ou le secret de Tolède erróneamente se refería a ella como obra de la colección Zuloaga. De manera que no está claro qué finalidad tenían entonces sus preguntas, si es que no eran del todo retóricas. En cualquier caso, no parece que se hayan tenido en consideración. El único que se dio cuenta del problema que existía entre esas dos pinturas fue Ellis Waterhouse en 1930, tal y como comprobamos en las anotaciones que hace al margen de la ficha de Cossío sobre la obra "de Zuloaga": "? But see Napoli Nobilissima, 1923"36.

En efecto, la obra napolitana no formaba parte del corpus del Greco, antes de su publicación por Valerio Mariani en $1953^{37}$ y de su catalogación como obra auténtica del maestro por Wethey

${ }^{30}$ Storm, 2011: 127, 130.

31 Wethey, 1962: 114, 118.

32 Hadjinicolaou, 1995: 600, n. 44. Cfr. también la ficha relativa a la pintura en Hadjinicolaou, 1995c: 336.

33 Álvarez Lopera, 2007: 89-90. Álvarez Lopera niega la procedencia de la colección de don Pedro Salazar de Mendoza.

34 "Da libri e periodici", Napoli Nobilissima, III (in copertina: ), 1923 (en portada: 1921): 32. La pintura tiene en el reverso las, muy posiblemente posteriores, inscripciones: "sono del Ill.mo Monsignor degli Oddi" e "Il Grecho".

${ }_{35}$ Mayer, 1911: 83 [“Paris, D. Ignazio Zuloaga, Hl. Franziskus, um 1577’], cfr. Mayer, 1921: 13 [Zuloaga, Parigi].

${ }^{36}$ Hadjinicolaou, 1999: 90-91.

${ }^{37}$ Mariani, 1953; 1956: 10. Sobre la obra véase también Pallucchini, 1981: 257. Caputi/Penta, 1985: 11. Hadjinicolaou, 1995b: 334-341 y 523-526. Álvarez Lopera, 2007: 90-91, con bibliografia precedente.

Arch. esp. arte, LXXXVIII, 352, OCTUBRE-DICIEMBRE 2015, 409-417

ISSN: 0004-0428, eISSN: 1988-8511, doi: 10.3989/aearte.2015.26 
en 1967. Este último, considerando la pintura napolitana de inferior calidad con respecto a la de la colección Zuloaga, rechazó, como un error, la pregunta - propuesta del autor anónimo de Napoli Nobilissima- "since two pictures exist and they differ in details"38.

Reiteramos que las dos citadas obras son muy similares entre sí, ya sea en la técnica pictórica y en la iconografía (basada en mayor parte en el grabado de Niccolò Boldrini a partir de una composición de Tiziano). Tienen además las mismas medidas, el mismo tipo de firma del artista y se consideran pinturas autógrafas del $\mathrm{Greco}^{39}$, fechadas en el periodo italiano, en los primeros años romanos ${ }^{40}$.

¿Sería, tal vez, legítimo hipotetizar que una de las dos obras tuviera que ver o incluso pudiera identificarse con la obra que en el siglo XVIII vio Amaduzzi en San Rocco en Roma que, con toda probabilidad tendría la misma iconografía y eventualmente las mismas medidas?

No podemos dar una respuesta válida a esta pregunta si no es cayendo en conjeturas, más o menos legítimas. Señalamos sobre todo que la procedencia de las dos pinturas permanece aún un poco oscura. Lopera señaló lo imprecisas que eran las noticias dadas por Cossío sobre la procedencia de la obra perteneciente a Zuloaga, mientras que el origen de la pintura napolitana aún permanece vaga, salvo la aparente información precisa de su compra recogida brevemente en Napoli Nobilissima ("hace años a un chamarilero en Nápoles").

Entonces, ¿queremos saber si es cierta la pregunta que se hacía el autor anónimo al identificar la pintura de la colección Pagliara con la reproducida en los libros de Barrés y Mayer? O quizás, siendo retórica, ¿escondía información? Lo mismo en relación con el préstamo de la pintura de Pagliara a Zuloaga que, desde el momento en que este se supone que poseía una pintura idéntica y firmada del mismo modo por el pintor cretense, aún permanece imprecisa, tanto por el motivo ("para limpiar el cuadro de la suciedad y de algunos viejos barnices oxidados"), como por la duración del préstamo ("muerto Pagliara, el pintor español devolvió el cuadrito a sus herederos") ${ }^{41}$.

En relación con la pintura napolitana, Mariani escribía en 1953 "Al paisaje movido y prerromántico que emerge de la ventana del estudio en el que el miniaturista (Clovio) fue pintado por el Greco ... se refiere, el ambiente de esta escena de los estigmas, casi idéntica de la pintura que posee Ignacio Zuloaga, varias veces publicada, pero anterior, como parece, a esta obra de mayor importancia, si el propio Zuloaga acosar de cartas, en un cierto momento, los propietarios del cuadro que estamos ilustrando para intentar tenerlo en su propia colección" ${ }^{\text {"42 }}$.

Una observación más al respecto: cuando en 2003 se expuso en Nueva York la obra casi totalmente idéntica a la de Nápoles, ofreciendo una ocasión a los estudiosos de contemplarla de nuevo $^{43}$, se pudo comprobar cómo existía una pequeña diferencia con respecto a la de la colección Zuloaga, tal y como venía dicho hasta ahora pero basándose solo en fotografías: la firma del artista no es exactamente la misma: en vez de "EПOІЕІ" dice "ЕПОIH"

${ }^{38}$ Wethey, 1967: 118

${ }^{39}$ Marías plantea dudas sobre si la pintura napolitana puede estar incluso en el corpus del Greco (Marías, 1999: 52, n. 37).

${ }^{40}$ Como hemos visto (n. 26), Cossío pretende que la obra pertenezca a Zuloaga, probablemente para explicar su procedencia, como obra de los primeros años del Greco en España, hacia 1577. Evidentemente por el mismo motivo, Gudiol, en 1971, sostiene que la obra fue traída de Italia a España por el propio Greco (Gudiol, 1971: 28). Wethey, en cambio, considera que las dos obras son anteriores. (“ca. 1560", Wethey, 1967: 117, 118). Teniendo en cuenta criterios estilísticos y comparándolo, por ejemplo, con el Retrato de Giulio Clovio, no hay duda de que la datación del San Francisco oscila entre finales del periodo veneciano del Greco y los primeros años de su estancia en Roma.

${ }^{41}$ Álvarez Lopera escribe que Pagliara prestó la pintura a Zuloaga hacia 1917-1918, algo que no puede ser porque el coleccionista napolitano murió en 1914 (Álvarez Lopera, 2007: 91).

${ }^{42}$ Mariani, 1953: 346.

${ }^{43}$ Así en 2007 Álvarez Lopera consideraba la pintura napolitana como "sólo una repetición, hasta cierto punto descuidada, de la versión Zuloaga" (Álvarez Lopera, 2007: 90).

${ }^{44}$ Davies intenta explicar la anomalía como un tipo raro usado por el Greco, aunque solo lo demuestra con un ejemplo, el retrato di Giulio Clovio (Davies, 2003: 295).

Arch. esp. arte, LXXXVIII, 352, OCTUBRE-DICIEMBRE 2015, 409-417 ISSN: 0004-0428, eISSN: 1988-8511, doi: 10.3989/aearte.2015.26 
Aunque si bien solo podemos seguir argumentando basándonos en hipótesis, debemos llegar a la conclusión de que no es fácil demostrar (aunque me parezca muy probable) que exista una relación directa entre la obra que una vez existía en la iglesia romana de San Rocco y las pinturas de San Francisco realizadas por el Greco durante su periodo romano actualmente conocidas. No por ello sería inútil tener en cuenta, en este sentido, que las circunstancias suscitadas en relación con las dos obras de San Francisco, ocurren precisamente a principios del siglo XX, en una época de gran interés por la obra del Greco y de importante intercambio y circulación de sus obras ${ }^{45}$.

\section{BIBLIOGRAFÍA}

Álvarez Lopera, José (1999): “De historiografía. La recuperación del período italiano del Greco". En: Hadjinicolaou, Nicos (ed.), El Greco in Italy and Italian Art. Proceedings of the International Symposium, Crete, 22-24 September 1995. Rethymno: University of Crete, pp. 23-46.

Álvarez Lopera, José (2007): El Greco: estudio y catálogo, volumen 2, tomo 1: Catálogo de obras originales. Creta, Italia, retablos y grandes encargos en España. Madrid: Fundación Arte Hispánico.

Barrès, Maurice (1912): Greco ou le secret de Tolède. París: Emile-Paul.

Barrès, Maurice (1923): Greco ou le secret de Tolède. París: Librairie Plon.

Berchet, Jean-Claude (1999): “Chateaubriand, Séroux D'Agincourt et les arts du Moyen Âge”. En: Fumaroli, M. (ed.), Chateaubriand et les Arts. París: Éditions de Fallois, pp. 57-81.

Caputi, Anna / Penta, Maria Teresa (1985): La raccolta d'arte della Fondazione Pagliara. Nápoles: Istituto "Suor Orsola Benincasa".

Carriero, Marina (1974): "Cultura e religione nel Settecento italiano: Giovanni Cristofano Amaduzzi e Scipione de' Ricci”. En: Rivista di Storia della Chiesa in Italia, XXVII, 1, Roma, pp. 94-126.

Carriero, Marina (1976): "Cultura e religione nel Settecento italiano: Giovanni Cristofano Amaduzzi e Scipione de' Ricci”. En: Rivista di Storia della Chiesa in Italia, XXX, 2, Roma, pp. 405-437.

Cassou, Jean (1931): Le Gréco. París: Rieder.

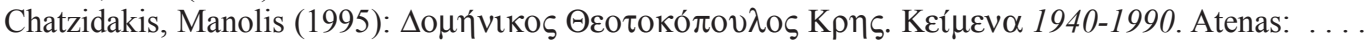

Cossío, Manuel B. (1908): El Greco. Madrid: Victoriano Suárez.

Davies, Davis (ed.) (2003): El Greco (cat. expo.). Nueva York: The Metropolitan Museum of Art - Londres: The National Gallery, 2003-2004.

Escholier, Raymond (1937): Greco. París: Librairie Floury.

Fabi, Angelo (1961): "Giancristofano Amaduzzi”. En: Dizionario Biografico degli Italiani. Roma: Treccani, vol. II, pp. 612-615.

Galavaris, George (1995): “El Greco's Image of St. Francis of Assisi”. En: Hadjinicolaou, Nicos (ed.), El Greco of Crete. Proceedings of the International Symposium. Iraklion, Crete, 1-5 September 1990, Iraklio, pp. 383-396.

Garofalo, Fausto (1949): L'Ospedale di San Rocco delle portorocuti e delle celate. Roma: Desclée \& C.

Griener, Pascal (1997): "La fatale attraction du Moyen Age. Jean-Baptiste Séroux d'Agincourt et l' 'Histoire de l'art par les monumens' (1810-1823)". En: Zeitschrift für Schweizerische Archäologie und Kunstgeschichte, LIV, 2, Zúrich, pp. 225-234.

Gudiol, José (1971): El Greco 1541-1614. Barcelona: Ediciones Polígrafa.

Hadjinicolaou, Nicos (1995a): "Une vision mystique du Greco: Barrès et le secret le Tolède". En: Hadjinicolaou, Nicos (ed.), El Greco of Crete. Proceedings of the International Symposium. Iraklion, Crete, 15 September 1990. Iraklio, pp. 599-611.

${ }^{45}$ No hay que olvidar el clima de misticismo de fuerte impacto a finales del siglo XIX y principio del siglo XX, que había provocado un gran interés -“culto" lo define Hadjnicolaou- por la figura de Francisco de Asís, Cfr. Hadjinicolaou, 1995a: 596-598.

Arch. esp. arte, LXXXVIII, 352, oCTUBRE-DICIEMBRE 2015, 409-417

ISSN: 0004-0428, eISSN: 1988-8511, doi: 10.3989/aearte.2015.26 


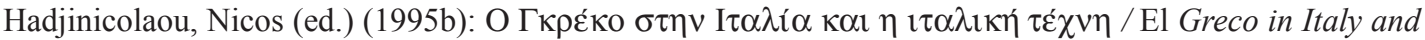
Italian Art, cat. expo. (Atenas, Pinacoteca Nacional), Atenas: Institute for Mediterranean StudiesFORTH.

Hadjinicolaou, Nicos (1999): "El Greco's Italian Period and Ellis K. Waterhouse". En: Hadjinicolaou, Nicos (ed.), El Greco in Italy and Italian Art. Proceedings of the International Symposium, Crete, 22-24 September 1995. Rethymno: University of Crete, pp. 71-103.

Haskell, Francis (1989): “Gibbon e la storia dell'arte”. En: Le metamorfosi del gusto. Studi su arte e pubblico nel XVIII e XIX secolo. Turín: Bollati Boringhieri, pp. 38-39.

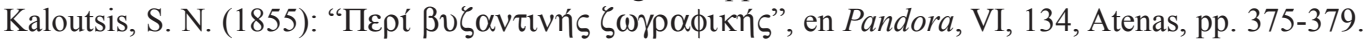

Lafond, Paul (1908): "La Collection de M. Ignacio Zuloaga". En: Les Arts, 74. París, pp. 22-31.

Lafond, Paul (1910): Paul Lafond, Le Greco. París: E. Sansot.

Legendre, M. / Hartmann, A. (1937): Domenikos Theotokopoulos Called El Greco. París: Éditions Hypérion.

Magnoni, Valentina (1931): Il Greco. Florencia: Casa Editrice "Memi".

Mariani, Valerio (1953): 'Le 'Stimmate di San Francesco' del Greco a Napoli”. En: Rivista dell'Istituto Nazionale d'archeologia e storia dell'arte, II. Roma, pp. 344-352.

Mariani, Valerio (1956): Fondazione Pagliara. La raccolta d'arte. Nápoles: Giannini.

Marías, Fernando (1999): “El Greco y los artistas de Italia: Venecia 1567-1570”. En: Hadjinicolaou, Nicos (ed.), El Greco in Italy and Italian Art. Proceedings of the International Symposium, Crete, 2224 September 1995. Rethymno: University of Crete, pp. 47-66.

Marías, Fernando (2009): “Luces y sombras de una pasión: Zuloaga y El Greco". En: Cuadernos de arte de la Universidad de Granada, XL, Granada, pp. 317-352.

Marías, Fernando (2014): El Greco. Life and Work. A New History. Londres: Thames and Hudson.

Mauclair, Camille (1931): Le Greco. París: Henri Laurens Éditeur.

Mayer, August L. (1911): El Greco. Múnich: Delphin Verlag.

Mayer, August L. (1916): El Greco. Múnich: Delphin Verlag.

Mayer, August L. (1921): Il Greco. Roma: Biblioteca d'arte illustrata.

Miarelli Mariani, Ilaria (2002): “Jean Baptiste Séroux d'Agincourt e la nascita della storia dell'arte medievale". En: Ricerche di storia dell'arte, 77, Roma, pp. 5-23.

Miarelli Mariani, Ilaria (2005): Séroux d'Agincourt e l'histoire de l'art par les monumens : riscoperta del Medioevo, dibattito storiografico e riproduzione artistica tra fine XVIII e inizio XIX secolo. Roma: Bonsignori.

Mondini, Daniela (2005): Mittelalter im Bild: Séroux d'Agincourt und die Kunsthistoriographie um 1800. Zúrich: Zurich InterPublishers.

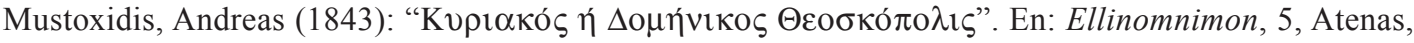
pp. 271-276.

Orlandi, Pellegrino Antonio (1753): Abecedario pittorico ... accresciuto da Pietro Guarienti. Venecia: Giambatista Pasquali.

Pacheco, Francisco (1990): El Arte de la pintura [Sevilla, 1649, edición, introducción y notas de Bonaventura Bassegoda i Hugas]. Madrid: Cátedra.

Pagano, G. (1831): Guida per le gallerie del museo Reale Borbonico. Nápoles: Torchi dell'osservatore Medico.

Pallucchini, Rodolfo (1981) (ed.): Da Tiziano a El Greco. Per la storia del manierismo a Venezia [cat. expo. (Venecia, Palazzo Ducale)]. Milán: Electa.

Pfister, Kurt (1941): El Greco, Viena, Gallus Verlag.

Pommier, Édouard (1997): "Le caractère des temps". En: Pommier, Édouard (ed.), Histoire de l'histoire de l'art, vol. II: XVIIIe et XIXe siècles. París: Musée du Louvre - Klincksieck, pp. 27-35.

Posterla, Francesco (1707): Roma sacra e moderna. Roma: Francesco Gonzaga.

Previtali, Giovanni (1989): La fortuna dei primitivi. Dal Vasari ai neoclassici [introducción Enrico Castelnuovo]. Turín: Einaudi.

Ricci, Corrado (1913): “Un quadretto del Greco a Bergamo”. En: Bollettino d'arte, VIII, Roma, pp. 309314.

Salerno, Luigi / Spagnesi, Gianfranco (1962): La chiesa di San Rocco all'Augusteo. Roma: Desclée \& C.

Arch. esp. arte, LXXXVIII, 352, OCTUBRE-DICIEMBRE 2015, 409-417 ISSN: 0004-0428, eISSN: 1988-8511, doi: 10.3989/aearte.2015.26 
Sargent, John S. (1916): "Foreward”. En Exhibition of Paintings by Ignacio Zuloaga 1916-1918, Nueva York, Redfield - Kendrick - Odell.

Séroux D'Agincourt, Jean Baptiste Louis Georges (1823): Histoire de l'art par les monumens depuis sa décadence au IVe siècle jusqu'à son renouvellement au XVIe siècle, vol. II: sculpture - peinture, París: Treuttel et Würtz.

Séroux D'Agincourt, Jean Baptiste Louis Georges (1835): Storia dell'arte col mezzo dei documenti dalla sua decadenza nel IV secolo fino al suo risorgimento nel XVI, con aggiunte italiane. Milán: Per Ranieri Fanfani, vol. V.

Storm, Eric (2011): El descubrimiento del Greco. Nacionalismo y arte moderno (1860-1914). Madrid: Fundación El Greco 2014 - Centro de Estudios Europa Hispánica - Marcial Pons.

Thiemann, Birgit (2012): "'Vive Greco et Goya!": Ignacio Zuloaga als Sammler und Vermittler". En: Wismer, Beat /Scholz-Hänsel, Michael (2012) (ed.): El Grego und die Moderne, cat. expo. (Düsseldorf, Museum Kunstpalast), Ostfildern, Hatje Cantz, pp. 374-385.

Titi, Filippo (1675): Studio di pittura, scoltura, \& architettura nelle chiese di Roma. Roma - Macerata: Giuseppe Piccini.

Titi, Filippo (1686): Ammaestramento utile, e curioso di pittura scoltura et architettura nelle chiese di Roma, Palazzi Vaticano, di Monte Cavallo, et altri, che s'incontrano nel cammino facile, che si fà per ritrovarle..., Roma: Giuseppe Vanacci.

Titi, Filippo (1763): Descrizione delle pitture, sculture e architetture esposte al pubblico in Roma opera cominciata dall'abate Filippo Titi da città di Castello, con l'aggiunta di quanto è stato fatto di nuovo fino all'anno presente. Roma: Marco Pagliarini.

Venuti, Ridolfino (1767): Roma moderna, Roma, Carlo Barbibelini.

Wethey, Harold E. (1962): El Greco and his School. Princeton-New Jersey: Princeton University Press.

Willumsen, J.-F. (1927): La jeunesse du peintre El Greco. Essai sur la transformation de l'artiste byzantin en peintre européen. París: G. Crès.

Fecha de recepción: 30-I-2015

Fecha de aceptación: 22-IV-2015

Arch. esp. arte, LXXXVIII, 352, OCTUBRE-DICIEMBRE 2015, 409-417

ISSN: 0004-0428, eISSN: 1988-8511, doi: 10.3989/aearte.2015.26 